\title{
Association between Anti-Mullerian Hormone and Ovarian Response and Pregnancy in Polycystic Ovary Syndrome Patients
}

\author{
Nayereh Ghomian $^{1 * \mathbb{D}}$, Marzieh Lotfalizadeh $^{1}$, Malihe Afiat $^{1}$, Farideh Golhasani Keshtan ${ }^{2}$
}

\begin{abstract}
Objectives: Polycystic ovary syndrome (PCOS) is considered as one of the causes of infertility. Anti-Mullerian hormone (AMH) is 2-3 times higher in patients with the PCOS compared to others. The present study aimed to survey the association between basal AMH levels and ovarian response in infertile PCOS patients.

Materials and Methods: In this cross-sectional study, 70 infertile PCOS women were enrolled referring to Milad Infertility Center (Mashhad, Iran) from May 2011 to April 2012. The basal blood level of AMH was evaluated. The patients received 5 mg of letrozole from the fifth cycle day (for 5 days) and then received 150 units of gonal $\mathrm{F}$ in the form of the recombinant follicle-stimulating hormone (FSH) on the ninth day of the cycle. Five thousand units of human chorionic gonadotropin (hCG) were prescribed for the patients who had one follicle of $\geq 18 \mathrm{~mm}$. Then, they should have had intercourse 36 hours later. After ovulation induction (OI) on the 10th cycle day, the second serum sample of AMH was taken.

Results: Changes of AMH did not have a significant relationship with the ovarian response, namely, it was lower in patients with positive ovarian response compared to those without any ovarian response. In addition, the basal level of AMH in patients with a positive response $(3.91 \pm 2.14)$ had no significant difference with the secondary AMH $(3.27 \pm 2.39)$ after the OI $(P=0.19)$.

Conclusions: Generally, the AMH was not a predictor of ovarian response in PCOS patients and the amount of AMH reduced after the treatment. This effect was maybe related to letrozole and gonadotropin.

Keywords: Anti-Mullerian Hormone, Polycystic Ovary Syndrome, Ovulation Induction, Infertility, Letrozole
\end{abstract}

\section{Introduction}

Polycystic ovary syndrome (PCOS) is among the primary causes of infertility due to anovulation, with a prevalence rate of $4 \%-6 \%$ in women of reproductive age $(1,2)$.

Anti-Mullerian hormone (AMH) is a glycoprotein hormone secreted by the granulosa cells of the antral and preantral follicles $(3,4)$.

$\mathrm{AMH}$ level decreases throughout the reproductive period and becomes undetectable at the time of menopause (4). However, an increased level of AMH can be observed in females suffering from PCOS, which indicates the presence of a larger number of antral follicles in such women (5). This hormone is a practical indicator of ovarian reserve and can serve as a predictor of ovarian response in cycles of in vitro fertilization (IVF) (6).

Letrozole is an oral non-steroidal aromatase inhibitor which has been used for the treatment of breast cancer after surgery and ovarian stimulation since $2001(7,8)$. Aromatase is a cytochrome p450 enzyme that catalyzes the conversion of androgens to the estrogens (7). Some studies have come up with conflicting results regarding the use of $\mathrm{AMH}$ as a predictor of ovarian response in patients suffering from PCOS $(9,10)$.
A number of studies have monitored changes in $\mathrm{AMH}$ level during IVF cycles involving either GnRH agonist or GnRH antagonist (11-15). However, no data is available concerning the possible relationship between the changes in AMH level and ovarian response in infertile women suffering from PCOS who have undergone ovulation induction with letrozole and follicle stimulating hormone (FSH).

It is suggested that AMH serum level can serve to predict the ovarian response and the ovarian hyperstimulation syndrome (OHSS) during implementing assisted reproduction technique (ART) for infertile women (11-15).

However, the exact mechanism regulating the AMH level during human folliculogenesis has remained extensively unknown. Several studies proposed that AMH level plays a pathological role during the process of folliculogenesis in PCOS (16-18). Lee et al, for instance, investigated patients with breast cancer who received ovulatory stimulation using letrozole and FSH and reported that the AMH level and the number of antral follicles could predict the result of embryo/oocyte cryopreservation. Nonetheless, the subjects of this study did not suffer from PCOS (12).

Received 23 December 2017, Accepted 10 June 2018, Available online 20 June 2018

${ }^{1}$ Department of Obstetrics and Gynecology, Neonatal and Maternal Research Center, Mashhad University of Medical Sciences, Mashhad, Iran ${ }^{2}$ Research Administrative Assistant, Mashhad University of Medical Sciences, Mashhad, Iran.

*Corresponding Author: Nayereh Ghomian, Tel: +989151101435, Email: ghomiann@mums.ac.ir 
Controversial studies exist regarding measuring the $\mathrm{AMH}$ level as a predictor of ovarian response in women with PCOS $(9,10)$.

The current study aimed at investigating the relationship between basal AMH levels and its changes and ovarian response in infertile PCOS patients in ovulation induction (OI) cycles by using letrozole and FSH.

\section{Materials and Methods}

Seventy infertile women with PCOS who referred to Milad Infertility Centre of Mashhad (during May 2011 to April 2012) were included in this cross-sectional research. This sample size was calculated based on a standard deviation of $2.06, d=0.5, \alpha=0.05$, and $\beta=0.8$. Rotterdam criteria were used to select these patients using convenient sampling technique. The PCOS was diagnosed when $\geq 2$ through the following three criteria: oligomenorrhea or amenorrhea, clinical hyperandrogenism or hyperandrogenemia, and polycystic ovaries on ultrasonography. All the patients were infertile (i.e., lack of pregnancy after one year of unprotected intercourse) and afflicted with PCOS.

The inclusion criteria were women with previously diagnosed PCOS according to Rotterdam criteria, aged between 20-35 years, and having a body mass index (BMI) between $18-30 \mathrm{~kg} / \mathrm{m}^{2}$. The exclusion criteria encompassed women with infertility of any other etiology, exposed to the cytotoxic drug, pelvic radiation therapy, or suffering from renal or liver diseases.

Patients underwent a vaginal ultrasonography scan (probe $7.5 \mathrm{MHz}$, ultrasonic) on day 3 of the menstrual cycle and their blood samples were taken for basal AMH, luteinizing hormone (LH), and FSH assays. Further, they received $5 \mathrm{mg}$ of letrozole (letrofem, Iran hormone, Iran) for five days (from the fifth day of the cycle) followed by 150 units of gonal-F in the form of recombinant FSH (MerckSerono Italia) on the ninth day of the cycle because most of the patients had a history of failed induction ovulation cycles. They were controlled by transvaginal sonography at day 10 for determining the number and size of the follicles and evaluating the endometrial line.

Ultra-sonographic scanning was continued if had clinical indication. On day 10 of the cycle, the second serum sample was taken for AMH after the induction. If the patient had an $18 \mathrm{~mm}$ follicle on one ovary, it was considered a positive response, otherwise, it was regarded as a negative one. Furthermore, if there were more than four follicles in both ovaries, it was assumed as hyperstimulation. Moreover, $5000 \mathrm{IU}$ of human chorionic gonadotropin (hCG) (Pregnyl) was administered followed by an intercourse performed 36 hours later if the ovary response was positive. Serum hCG level was then measured two weeks later. However, hCG was not administered to the hyper-stimulated group.

Clinical pregnancy was defined as the presence of gestational sac with the embryo on ultrasonography performed at 7-8 weeks after the last menstrual period.
Two serum samples from each patient were stored at $-70^{\circ} \mathrm{C}$ until further analysis. AMH level was measured using a specific ELISA kit (Beckman Coulter, USA). The age, BMI, duration of fertility, ovarian response, the result of AMH level (basal and secondary), and pregnancy results were recorded for each patient.

Data were analyzed by SPSS (statistical package for the social sciences) software, version 16 . Inferential tests including Mann-Whitney, Kruskal-Wallis, $\chi^{2}$, and Pearson correlation coefficient were used for data analysis. The $P$ value $<0.05$ was considered statistically significant.

\section{Results}

Out of 70 patients, $12(17.1 \%)$ of them had a negative ovarian response while $50(71.4 \%)$ patients had an appropriate ovarian response. In addition, the remaining 8 (11.4\%) patients had hyperstimulation (i.e., $\geq 4$ follicles). A positive clinical pregnancy was found in 10 (14.3\%) of the patients. Multiple pregnancies were not observed.

The age, infertility duration, BMI, number of previous induction cycles, and serum FSH were not different between the three study groups (Table 1). Mean of AMH changes was lower in women with appropriate ovarian response (1-3 follicles) and hyperstimulation group ( $\geq 4$ follicles) compared to that of the no response group (Table 2). Age, BMI, FSH, and LH levels were not significantly associated with basal AMH although infertility duration had a significant relationship with basal AMH (Table 3). Basal and secondary AMH and its changes had no significant correlation with pregnancy (Table 4).

\section{Discussion}

The results of this study on 70 PCOS patients treated with letrozole and gonadotropin demonstrated that basal $\mathrm{AMH}$ was at a high level. The basal AMH level did not statistically vary in the group without an ovary response or with ovary response. This contradicts with the findings of many other studies. Kaya et al in their study found that serum AMH level on the third day of IVF cycle was a marker of the ovary response and fertility at the ART cycles in PCOS patients (19). However, most of the previous studies have been done in IVF cycles. However, El-Halawaty et al used clomiphene citrate in PCOS patients and indicated that basal AMH was a good predictive factor for ovary response (10).

Further, Lamazou et al aimed to compare the IVF results based on AMH level. Accordingly, the level of AMH was checked in 342 patients who were in the first IVF cycle. The result demonstrated that although AMH was a quantitative marker of the ovary response, it did not evaluate the quality of oocyte. Furthermore, the AMH serum level was not a predictive factor of pregnancy in IVF cycles (9). The reason for this finding in our study may be a less number of cases or the number of preantral and primary antral follicles that secreted AMH. The other cause could be the letrozole that led to an increase in 
Table 1. Age, Infertility Duration, BMI, Cycle Number, FSH and LH in 3 Different Ovarian Response Groups

\begin{tabular}{|c|c|c|c|c|}
\hline & \multicolumn{3}{|c|}{ Ovarian Response } & \multirow{2}{*}{$P$ Value } \\
\hline & None $(n=12)$ & 1-3 Follicles $(n=50)$ & $\geq 4$ Follicles $(n=8)$ & \\
\hline Age (y) & $26.75 \pm 5.22 *$ & $26.86 \pm 4.25$ & $25.77 \pm 3.75$ & 0.811 \\
\hline Infertility duration (y) & $7.41 \pm 5.22$ & $5.84 \pm 3.32$ & $5.62 \pm 2.73$ & 0.383 \\
\hline BMI $\left(\mathrm{kg} / \mathrm{m}^{2}\right)$ & $27.1 \pm 1.15$ & $26.76 \pm 2.70$ & $25.56 \pm 2.54$ & 0.533 \\
\hline Previous ovulation induction cycle (number) & $1.45 \pm 1.21$ & $1.12 \pm 0.97$ & $1.12 \pm 1.12$ & 0.622 \\
\hline $\mathrm{FSH}(\mathrm{UI} / \mathrm{L})$ & $6.89 \pm 2.84$ & $5.76 \pm 2.20$ & $5.42 \pm 2.70$ & 0.297 \\
\hline LH (UI/L) & $10.85 \pm 6.38$ & $7.68 \pm 6.89$ & $8.93 \pm 3.45$ & 0.01 \\
\hline
\end{tabular}

BMI: body mass index; FSH, follicle-stimulating hormone; $\mathrm{LH}$, luteinizing hormone.

* Mean \pm SD.

Statistical test: Analysis of variance and Kruskal-Wallis test.

Table 2. Basal and Secondary Serum AMH in 3 Different Ovarian Response Groups

\begin{tabular}{|c|c|c|c|c|}
\hline & \multicolumn{3}{|c|}{ Ovarian Response } & \multirow{2}{*}{$P$ Value } \\
\hline & None $(n=12)$ & 1-3 follicles $(n=50)$ & $\geq 4$ follicles $(n=8)$ & \\
\hline Basal AMH (ng/mL) & $4.47 \pm 3.91$ & $3.91 \pm 2.14$ & $4.51 \pm 2.47$ & 0.778 \\
\hline Secondary AMH (ng/mL) & $4.60 \pm 3.41$ & $3.27 \pm 2.39$ & $2.68 \pm 1.98$ & 0.193 \\
\hline $\mathrm{AMH}$ changes $(\mathrm{ng} / \mathrm{mL})$ & $0.125 \pm 3.14$ & $-.64 \pm 1.86$ & $-1.82 \pm 0.75$ & 0.124 \\
\hline
\end{tabular}

AMH: anti-Mullerian hormone.

* Mean \pm SD.

Statistical test: Analysis of variance.

androgen level, but a decrease in the effect of basal AMH on predicting the treatment response (9).

The AMH level was compared before and after the treatment in our study. The results revealed that the AMH level significantly decreased in all the patients. This decline was due to a decrease in AMH production in prominent follicles. However, such a decrease was observed in both patients with and without an ovary response. Interestingly, the more prominent was the follicle, the more decrease was observed in the AMH.

Considering that most of the cycles in this study were monofolicle, which was the same as the natural cycles in healthy women, a decrease of AMH contradicted this belief that AMH level was a plateau in the menstrual cycle. Therefore, this rise a question of whether such a result is due to different behavior of AMH in PCOS patients or this decrease is related to the letrozole effect on AMH concentration. This question is subject to further investigations.

In an article about a patient with breath cancer, the authors declared that AMH was a predictor of embryo and oocyte cryopreservation cycle outcomes which were stimulated with letrozole and FSH. Nonetheless, it should be noted that the study patient was not a case of PCOS (20).

Pilltonen et al reported that the AMH levels remained three-fold higher throughout the reproductive age in PCOS patients but decreased during treatment with metformin (21). However, Pellatt et al highlighted that there was a subgroup of women with PCOS who had an elevated level of AMH and were resistant to the OI. Thus, AMH was not the sole cause of anovulation (22). To the best of our knowledge, no study is available regarding the effect of letrozole on AMH concentration in PCOS patients. The precise regulatory mechanism of $\mathrm{AMH}$ is unclear. However, a pathologic role in PCOS has been suggested by some studies (16-18).

There was no significant relationship between AMH level and other factors including age, BMI and FSH, and

Table 3. The Correlation of Age, Infertility Duration, BMI, FSH, LH with Basal Serum AMH Concentration

\begin{tabular}{lcc}
\hline & \multicolumn{2}{c}{ Basal AMH } \\
\cline { 2 - 3 } & $\boldsymbol{r}$ & $\boldsymbol{P}$ Value \\
\hline Age (y) & 0.043 & 0.726 \\
Infertility duration (y) & 0244 & 0.042 \\
BMI (kg/m) & 0.050 & 0.685 \\
FSH (IU/L) & 0.074 & 0547 \\
LH (IU/L) & -0.101 & 0407 \\
\hline
\end{tabular}

$\mathrm{AMH}$, anti-Mullerian hormone; FSH, Follicle-stimulating hormone; $\mathrm{LH}$ luteinizing hormone; BMI, body mass index.

$r$ : Pearson correlation coefficient.

Table 4. The correlation of Basal, Secondary and Changes of Serum AMH Concentration With Pregnancy

\begin{tabular}{lccc}
\hline & \multicolumn{2}{c}{ Pregnancy } & \multirow{2}{*}{ P Value } \\
\cline { 2 - 3 } & No $(\mathrm{n}=60)$ & Yes $(\mathrm{n}=10)$ & \\
\hline Basal AMH $(\mathrm{ng} / \mathrm{mL})$ & $4.113 .11 \pm^{*}$ & $3.872 .44 \pm$ & 0.953 \\
Secondary AMH $(\mathrm{ng} / \mathrm{mL})$ & $3.442 .73 \pm$ & $3.371 .46 \pm$ & 0.475 \\
AMH Changes $(\mathrm{ng} / \mathrm{mL})$ & $-0.672 .19 \pm$ & $-0.501 .36 \pm$ & 0.673 \\
\hline
\end{tabular}

$\mathrm{AMH}$ : anti-Mullerian hormone.

* Mean \pm SD.

Statistical test: Mann-Whitney. 
LH level. However, infertility duration was the only factor which was significantly associated with the AMH level. This could be due to a high level of AMH in women with severe PCOS, which led to more resistance to treatment. In the relationship between age, BMI, LH and FSH levels, infertility duration, and quantity of previous treatment cycles and the ovarian response, only LH was found to have a significant effect being reported by previous studies $(10,19,23)$.

The present results demonstrated that serum AMH level decreased during letrozole treatment. While our study was the first one to indicate that AMH was not a useful marker for predicting the ovarian response in OI cycles with letrozole and FSH in PCOS patients, larger studies are needed to investigate the value of $\mathrm{AMH}$ as a predictor of ovarian response in PCOS patients. One of the limitations of this study was the dropout of two patients. However, it could not adjust the results.

\section{Conclusions}

Finally, it can be concluded that the basal AMH was not a predictive factor for ovary response in PCOS patient. Moreover, AMH level decreased significantly before and after the treatment regardless of the ovary response that could be due to the effect of letrozole and gonadotropin. Further research is necessary to elucidate the mechanism of $\mathrm{AMH}$ changes during the OI with letrozole and recombinant FSH.

\section{Conflict of Interests}

Authors declare that they have no conflict of interests.

\section{Ethical Issues}

All the patients were asked to fill the written informed consent form. This study was approved by the Ethics Committee of Mashhad University of Medical Sciences. The reference code obtained was IR.MUMS.REC.1390.31.

\section{Financial Support}

This study was supported by Vice Chancellor for Research of Mashhad University of Medical Sciences.

\section{Acknowledgments}

This manuscript was written based on Dr. Rikhtegaran's special thesis (with a code of 2657-T) recorded in Mashhad University of Medical Sciences. We kindly appreciate the cooperation of the infertility unit personnel of Milad Centre and also the patients who took part in this study. Additionally, Special thanks go to the Vice Chancellor for Research Department at Mashhad University of Medical Sciences for their support.

\section{References}

1. Boivin J, Bunting L, Collins JA, Nygren KG. International estimates of infertility prevalence and treatment-seeking: potential need and demand for infertility medical care.
Hum Reprod. 2007;22(6):1506-1512. doi:10.1093/humrep/ dem046

2. Wilkes S, Chinn DJ, Murdoch A, Rubin G. Epidemiology and management of infertility: a population-based study in UK primary care. Fam Pract. 2009;26(4):269-274. doi:10.1093/fampra/cmp029

3. Salmassi A, Mettler L, Hedderich J, et al. Cut-Off Levels of Anti-Mullerian Hormone for The Prediction of Ovarian Response, In Vitro Fertilization Outcome and Ovarian Hyperstimulation Syndrome. Int J Fertil Steril. 2015;9(2):157-167. doi:10.22074/ijfs.2015.4236

4. Kriseman M, Mills C, Kovanci E, Sangi-Haghpeykar H, Gibbons W. Antimullerian hormone levels are inversely associated with body mass index (BMI) in women with polycystic ovary syndrome. J Assist Reprod Genet. 2015;32(9):1313-1316. doi:10.1007/s10815-015-0540-0

5. Tremellen K, Zander-Fox D. Serum anti-Mullerian hormone assessment of ovarian reserve and polycystic ovary syndrome status over the reproductive lifespan. Aust N Z J Obstet Gynaecol. 2015;55(4):384-389. doi:10.1111/ ajo. 12366

6. Aghssa MM, Tarafdari AM, Tehraninejad ES, et al. Optimal cutoff value of basal anti-mullerian hormone in iranian infertile women for prediction of ovarian hyper-stimulation syndrome and poor response to stimulation. Reprod Health. 2015;12:85. doi:10.1186/s12978-015-0053-4

7. El-Gharib MN, Mahfouz AE, Farahat MA. Comparison of letrozole versus tamoxifen effects in clomiphen citrate resistant women with polycystic ovarian syndrome. J Reprod Infertil. 2015;16(1):30-35.

8. Rasekhjahromi A, Maalhagh M, Hosseinpoor M, Farhang $\mathrm{H}$, Alavi F. A clomiphene citrate and letrozol varsus tamoxifen and letrozole as an infertility treatment in women with polycystic ovary syndrome. Pak J Biol Sci. 2015;18(6):300. doi:10.3923/pjbs.2015.300.303

9. Lamazou F, Genro V, Fuchs F, et al. [Serum AMH level is not a predictive value for IVF in modified natural cycle: analysis of 342 cycles]. J Gynecol Obstet Biol Reprod (Paris). 2011;40(3):205-210. doi:10.1016/j.jgyn.2011.02.002

10. El-Halawaty S, Rizk A, Kamal M, et al. Clinical significance of serum concentration of anti-Mullerian hormone in obese women with polycystic ovary syndrome. Reprod Biomed Online. 2007;15(5):495-499.

11. Nakhuda GS, Chu MC, Wang JG, Sauer MV, Lobo RA. Elevated serum mullerian-inhibiting substance may be a marker for ovarian hyperstimulation syndrome in normal women undergoing in vitro fertilization. Fertil Steril. 2006;85(5):1541-1543. doi:10.1016/j.fertnstert.2005.10.052

12. Lee JR, Kim SH, Kim SM, et al. Anti-Mullerian hormone dynamics during controlled ovarian hyperstimulation and optimal timing of measurement for outcome prediction. Hum Reprod. 2010;25(10):2597-2604. doi:10.1093/ humrep/deq204

13. Gnoth C, Schuring AN, Friol K, Tigges J, Mallmann P, Godehardt E. Relevance of anti-Mullerian hormone measurement in a routine IVF program. Hum Reprod. 2008;23(6):1359-1365. doi:10.1093/humrep/den108

14. Fanchin R, Mendez Lozano DH, Frydman N, et al. AntiMullerian hormone concentrations in the follicular fluid of the preovulatory follicle are predictive of the implantation potential of the ensuing embryo obtained by in vitro fertilization. J Clin Endocrinol Metab. 2007;92(5):1796- 
1802. doi:10.1210/jc.2006-1053

15. Fanchin R, Mendez Lozano DH, Louafi N, AchourFrydman N, Frydman R, Taieb J. Dynamics of serum anti-Mullerian hormone levels during the luteal phase of controlled ovarian hyperstimulation. Hum Reprod. 2005;20(3):747-751. doi:10.1093/humrep/deh669

16. Zadehmodarres S, Heidar Z, Razzaghi Z, Ebrahimi L, Soltanzadeh K, Abed F. Anti-mullerian hormon level and polycystic ovarian syndrome diagnosis. Iran J Reprod Med. 2015;13(4):227-230.

17. Weenen C, Laven JS, Von Bergh AR, et al. Anti-Mullerian hormone expression pattern in the human ovary: potential implications for initial and cyclic follicle recruitment. Mol Hum Reprod. 2004;10(2):77-83.

18. Pigny P, Merlen E, Robert Y, et al. Elevated serum level of anti-mullerian hormone in patients with polycystic ovary syndrome: relationship to the ovarian follicle excess and to the follicular arrest. J Clin Endocrinol Metab. 2003;88(12):5957-5962. doi:10.1210/jc.2003-030727

19. Kaya C, Pabuccu R, Satiroglu H. Serum antimullerian hormone concentrations on day 3 of the in vitro fertilization stimulation cycle are predictive of the fertilization, implantation, and pregnancy in polycystic ovary syndrome patients undergoing assisted reproduction. Fertil Steril. 2010;94(6):2202-2207. doi:10.1016/j.fertnstert.2009.12.002

20. Ocal P, Sahmay S, Cetin M, Irez T, Guralp O, Cepni I. Serum anti-Mullerian hormone and antral follicle count as predictive markers of OHSS in ART cycles. J Assist Reprod Genet. 2011;28(12):1197-1203. doi:10.1007/s10815-0119627-4

21. Piltonen T, Morin-Papunen L, Koivunen R, Perheentupa A, Ruokonen A, Tapanainen JS. Serum anti-Mullerian hormone levels remain high until late reproductive age and decrease during metformin therapy in women with polycystic ovary syndrome. Hum Reprod. 2005;20(7):18201826. doi:10.1093/humrep/deh850

22. Pellatt L, Rice S, Mason HD. Anti-Mullerian hormone and polycystic ovary syndrome: a mountain too high? Reproduction. 2010;139(5):825-833. doi:10.1530/rep-090415

23. Broer SL, Mol BW, Hendriks D, Broekmans FJ. The role of antimullerian hormone in prediction of outcome after IVF: comparison with the antral follicle count. Fertil Steril. 2009;91(3):705-714. doi:10.1016/j.fertnstert.2007.12.013

(c) 2019 The Author (s); This is an open-access article distributed under the terms of the Creative Commons Attribution License (http://creativecommons.org/licenses/by/4.0), which permits unrestricted use, distribution, and reproduction in any medium, provided the original work is properly cited. 